\title{
Pharmacokinetics of a novel endectoparasiticide topical formulation for cats, combining esafoxolaner, eprinomectin and praziquantel
}

\author{
Virginie Jacquot ${ }^{1}$, Prescillia Buellet ${ }^{1}$, Laura Letendre ${ }^{2}$, Wei Tong ${ }^{2}$, Henry Li $^{2}$, and Eric Tielemans ${ }^{1, *}$ \\ ${ }^{1}$ Boehringer-Ingelheim Animal Health, 29 avenue Tony Garnier, 69007 Lyon, France \\ ${ }^{2}$ Boehringer-Ingelheim Animal Health, 631 Route 1, North Brunswick, NJ 08902, USA
}

Received 6 May 2020, Accepted 8 March 2021, Published online 2 April 2021

\begin{abstract}
Esafoxolaner, a purified enantiomer of afoxolaner with insecticidal and acaricidal properties, is combined with eprinomectin and praziquantel in NexGard ${ }^{\circledR}$ Combo, a novel topical endectoparasiticide formulation for cats. The parasiticide potencies of topical esafoxolaner, eprinomectin and praziquantel, are based on transcutaneous absorption, systemic distribution, and exposure of respective target parasites. For each compound, the pharmacokinetic profile, non-interference, dose linearity/proportionality after one administration, and the accumulation and time to reach a steady state after repeated monthly administrations of the novel formulation, were investigated. After one topical application of NexGard ${ }^{\circledR}$ Combo at the minimum recommended dose, the mean plasma concentration of esafoxolaner immediately reached (and remained at) a level supporting rapid onset and sustained efficacy against ectoparasites for at least 1 month. The mean $C_{\max }, T_{\max }, T_{1 / 2}$, and the topical bioavailability of esafoxolaner were $130 \mathrm{ng} / \mathrm{mL}, 7.1$ days, 21.7 days and $47.2 \%$, respectively, and the plasma profiles of eprinomectin and praziquantel supported their known endoparasiticide properties. No relevant interference between the three compounds was observed. Dose proportionality was demonstrated for the three compounds over a range of $0.5 \times$ to $2 \times$ the minimum recommended dose. Steady state after repeated monthly administrations was reached by the second dose for praziquantel and by the fifth dose for esafoxolaner and eprinomectin. Accumulation was limited and drug plasma concentrations were maintained within a safe level.
\end{abstract}

Key words: Cat, Esafoxolaner, Eprinomectin, Praziquantel, Topical, Pharmacokinetics.

Résumé - Pharmacocinétique d'une nouvelle formulation topique d'endectoparasiticide pour chats, combinant esafoxolaner, éprinomectine et praziquantel. L'esafoxolaner, un énantiomère purifié d'afoxolaner aux propriétés insecticides et acaricides, est combiné à l'éprinomectine et au praziquantel dans NexGard ${ }^{\circledR}$ Combo, une nouvelle formulation endectoparasiticide topique pour chats. Les pouvoirs parasiticides de l'esafoxolaner topique, de l'éprinomectine et du praziquantel sont basés sur l'absorption transcutanée, la distribution systémique et l'exposition des parasites cibles respectifs. Pour chaque composé, le profil pharmacocinétique, la non-interférence, la linéarité/ proportionnalité de dose après une administration, ainsi que l'accumulation et le temps nécessaire pour atteindre un état d'équilibre après des administrations mensuelles répétées de la nouvelle formulation, ont été étudiés. Après une application topique de NexGard ${ }^{\circledR}$ Combo à la dose minimale recommandée, la concentration plasmatique moyenne d'esafoxolaner a immédiatement atteint et est restée à un niveau soutenant une apparition rapide et soutenue de l'efficacité contre les ectoparasites pendant au moins un mois. La $C_{\max }$ moyenne, la $T_{\max }$, la $T_{1 / 2}$, et la biodisponibilité topique de l'esafoxolaner était respectivement de $130 \mathrm{ng} / \mathrm{mL}, 7,1$ jours, 21,7 jours et 47,2\%, et les profils plasmatiques de l'éprinomectine et du praziquantel ont confirmé leurs propriétés endoparasiticides connues. Aucune interférence significative entre les trois composés n'a été observée. La proportionnalité de la dose a été démontrée pour les trois composés sur une plage de $0,5 \times$ à $2 \times$ la dose minimale recommandée. L'état d'équilibre après des administrations mensuelles répétées a été atteint par la deuxième dose de praziquantel et par la cinquième dose d'esafoxolaner et d'éprinomectine. L'accumulation était limitée et les concentrations plasmatiques du médicament étaient maintenues à un niveau sûr.

*Corresponding author: eric.tielemans@boehringer-ingelheim.com

Special Issue - NexGard ${ }^{\circledR}$ Combo (esafoxolaner, eprinomectin, praziquantel): A new endectocide spot-on formulation for cats. Invited Editor: Frédéric Beugnet

This is an Open Access article distributed under the terms of the Creative Commons Attribution License (https://creativecommons.org/licenses/by/4.0), which permits unrestricted use, distribution, and reproduction in any medium, provided the original work is properly cited. 


\section{Introduction}

Cats may be affected by multiple parasites, some of them with zoonotic potential $[1,17,21,38]$. NexGard ${ }^{\circledR}$ Combo, a novel topical endectoparasiticide formulation, combines esafoxolaner, eprinomectin, and praziquantel, and offers a new therapeutic solution to feline veterinary medicine. The parasiticide potency of the three active ingredients in this novel formulation is based on transcutaneous absorption, followed by systemic distribution, and exposure of their respective target parasites. The main objectives of treatment with this novel formulation are to kill existing fleas, ticks and/or ear mites, to prevent new infestations by fleas and/or ticks for at least 1 month, and to kill existing nematodes and cestodes. One topical application should therefore provide an adequate peak plasma concentration for the three active ingredients for rapid onset of activity, and for adequate plasma esafoxolaner levels for sustained efficacy for at least 1 month.

Esafoxolaner, a novel compound, is the purified and active (S)-enantiomer of afoxolaner, a racemic compound from the isoxazoline class. Afoxolaner is commercially available as an oral acaricide and insecticide for dogs, as a single active substance $\left(\mathrm{NexGard}^{\circledR}\right)$ [5] or in combination with milbemycin oxime (NexGard Spectra ${ }^{\circledR}$ ) [6], a macrocyclic lactone. The acaricidal efficacy of afoxolaner was tested off-label in cats against ear mites and was demonstrated efficacious at $2.5 \mathrm{mg} / \mathrm{kg}$ [22]. The advantage of using a purified and active enantiomer is to lower the dose of the active ingredient and consequently lower potential for side effects and chemical and pharmacological interactions (the acaricidal dose of esafoxolaner is $1.44 \mathrm{mg} / \mathrm{kg}$ in NexGard ${ }^{\circledR}$ Combo). Esafoxolaner acts as an antagonist at ligand-gated chloride channels, with high specificity for a unique binding site in insect and acarid gamma-aminobutyric acid (GABA)-gated chloride channels. It induces hyper-excitation with uncontrolled activity of the central nervous system and death of insects and acarids [26, 40]. There are no known relevant esafoxolaner binding sites in the mammalian GABA receptors; the selective toxicity of esafoxolaner against insects and acarids versus mammals may be inferred by the differential sensitivity of the arthropods' GABA receptors versus mammalian counterparts [26, 31]. Following oral administration in dogs with or without milbemycin oxime, the afoxolaner plasma profile shows rapid onset and sustained efficacy against ectoparasites for at least 1 month, with a rapid $T_{\max }$ and a long half-life $[19,20]$. There is also a high correlation between the afoxolaner plasma concentration in dogs and efficacy against fleas and ticks, and the presence of milbemycin oxime does not interfere with afoxolaner efficacy [19]. This was confirmed in clinical studies demonstrating high ectoparasiticide efficacies in dogs for both NexGard ${ }^{\circledR}$ and NexGard Spectra ${ }^{\circledR}[9-11,14,15,18$, 24, 25, 30].

Eprinomectin is an avermectin, from the macrocyclic lactone class, binding selectively to glutamate-gated chloride ion channels on nerves and muscular cells of several types of invertebrates, including nematodes. Eprinomectin is a well-known compound used in cattle, sheep and goats [27, 33], and cats $[12,29]$. Praziquantel is a pyrazino-isoquinoline derivative anthelminthic that acts specifically on cestodes and trematodes, and is a well-known compound in veterinary and human medicine, in oral and topical forms [2, 3, 13, 29, 32, 35, 39].

The pharmacokinetic (PK) profiles of eprinomectin and praziquantel are well-known in Broadline ${ }^{\circledR}$, a topical product for cats combining both active substances with the ectoparasiticide compounds fipronil and (S)-methoprene. Eprinomectin and praziquantel dosages, concentrations and volumes are identical in Broadline ${ }^{\circledR}$ and in NexGard ${ }^{\circledR}$ Combo and have been shown to lack in vivo absorption, distribution, metabolism and excretion (ADME) interactions in Broadline ${ }^{\circledR}$ [16].

Nevertheless, since in NexGard ${ }^{\circledR}$ Combo the three active ingredients are in a fixed novel combination, there is a potential for interaction, i.e. an effect on ADME, resulting in a modified PK profile for a specific active substance due to the presence of the other active susbtance(s). It is important to fully understand the relevant PK interactions of the active ingredients in a combination to identify any potential consequence on the efficacy and safety of the product.

Furthermore, as this novel formulation may be administered repeatedly and monthly, it is important to study the level of accumulation of each compound following repeated administration, as there is potential for baseline plasma levels to increase with consequences on efficacy and/or safety.

This manuscript describes the two studies that were carried out to investigate the pharmacokinetic properties of this novel formulation, in which the PK profile of the three compounds, their non-interference, their dose proportionality, and the accumulation effect and time to steady states were studied.

\section{Materials and methods \\ Ethics}

The study protocols were reviewed and approved by the Boehringer-Ingelheim Animal Health Inc. Institutional Animal Care and Use Committee (IACUC). Cats were managed and handled similarly and with due regard for their well-being.

\section{Compliance}

Both study designs followed EMEA/CVMP/133/99-FINAL Guidelines for the Conduct of Pharmacokinetic Studies in Target Animal Species, and were conducted in accordance with the Organization for Economic Co-operation and Development (OECD) Principles of Good Laboratory Practice (Revised 1997, issued Jan 1998) ENV/MC/CHEM(98)17.

The two studies were run in the same GLP-certified test facility.

\section{Animals, husbandry and health}

A total of 62 purpose-bred Domestic Short/Long-hair cats sourced from the same licensed breeder were used, 30 cats (15 males and 15 females) weighing 2.2-6.8 kg and aged 9-13 months in Study \#1, and 32 (16 males and 16 females) cats weighing 2.7-6.6 kg and aged 6-10 months in Study \#2. Each cat was uniquely identified with a microchip and acclimated to the study conditions for at least 10 days before 
Table 1. Study designs.

\begin{tabular}{|c|c|c|c|c|}
\hline Study/Group & & $\begin{array}{l}\text { Dosage } \\
(\mathrm{mL} / \mathrm{kg})\end{array}$ & $\begin{array}{l}\text { Treatment } \\
\operatorname{Day}(\mathrm{s})\end{array}$ & Blood collection days (minutes, hours after treatment) \\
\hline \multicolumn{5}{|c|}{ Study \#1 - Pharmacokinetics and non-interference } \\
\hline $\begin{array}{l}\text { NexGard }^{\circledR} \text { Combo - } \\
\text { Topical }^{1}\end{array}$ & 8 & 0.12 & 0 & $0(4 \mathrm{~h}, 8 \mathrm{~h}), 1(24 \mathrm{~h}, 32 \mathrm{~h}), 2,4,7,14,21,29,42,56,70$ \\
\hline $\begin{array}{l}\text { Esafoxolaner - } \\
\text { Topical }^{2}\end{array}$ & 8 & 0.12 & 0 & $0(4 \mathrm{~h}, 8 \mathrm{~h}), 1(24 \mathrm{~h}, 32 \mathrm{~h}), 2,4,7,14,21,29,42,56,70$ \\
\hline Broadline $^{\circledR}-$ Topical $^{3}$ & 8 & 0.12 & 0 & $0(4 \mathrm{~h}, 8 \mathrm{~h}), 1(24 \mathrm{~h}, 32 \mathrm{~h}), 2,4,7,14,21,29,42,56,70$ \\
\hline $\begin{array}{l}\text { Esafoxolaner - } \\
\text { Intravenous }^{2}\end{array}$ & 6 & 0.12 & 0 & 0 (15 min, $30 \mathrm{~min}, 2 \mathrm{~h}, 4 \mathrm{~h}, 8 \mathrm{~h}), 1(24 \mathrm{~h}, 32 \mathrm{~h}), 2,4,7,14,21,29,42,56,70$ \\
\hline \multicolumn{5}{|c|}{ Study \#2 - Dose proportionality and multiple-dose kinetics } \\
\hline $\begin{array}{l}\text { NexGard }{ }^{\circledR} \text { Combo - } \\
\text { Topical- } 0.5 \times^{4}\end{array}$ & 8 & 0.06 & 0 & $0(2 \mathrm{~h}, 4 \mathrm{~h}, 8 \mathrm{~h}), 1(24 \mathrm{~h}, 32 \mathrm{~h}), 2,4,7,14,21,28,42,56,70,84$ and 91 \\
\hline $\begin{array}{l}\text { NexGard }{ }^{\circledR} \text { Combo - } \\
\text { Topical- } 1 \times^{1}\end{array}$ & 8 & 0.12 & 0 & $0(2 \mathrm{~h}, 4 \mathrm{~h}, 8 \mathrm{~h}), 1(24 \mathrm{~h}, 32 \mathrm{~h}), 2,4,7,14,21,28,42,56,70,84$ and 91 \\
\hline $\begin{array}{l}\text { NexGard }{ }^{\circledR} \text { Combo - } \\
\text { Topical }-2 x^{5}\end{array}$ & 8 & 0.24 & 0 & $0(2 \mathrm{~h}, 4 \mathrm{~h}, 8 \mathrm{~h}), 1(24 \mathrm{~h}, 32 \mathrm{~h}), 2,4,7,14,21,28,42,56,70,84$ and 91 \\
\hline $\begin{array}{l}\text { NexGard }{ }^{\circledR} \text { Combo - } \\
\text { Topical- } 1 \times{ }^{1}\end{array}$ & 8 & 0.12 & $\begin{array}{c}0,28,56 \\
84,112\end{array}$ & $\begin{array}{l}0(2 \mathrm{~h}, 4 \mathrm{~h}, 8 \mathrm{~h}), 1(24 \mathrm{~h}, 32 \mathrm{~h}), 2,4,7,14,21,28^{*}, 56^{*}, 84^{*}, 112(0 \mathrm{~h} *, 2 \mathrm{~h}, 4 \mathrm{~h}, 8 \mathrm{~h}), 113 \\
\quad(24 \mathrm{~h}, 32 \mathrm{~h}), 114(48 \mathrm{~h}), 116,119,126,133,140,154 \text { and } 168\end{array}$ \\
\hline \multicolumn{5}{|c|}{$\begin{array}{l}{ }^{*} \text { Blood sampling before treatment. } \\
1 \text { Esafoxolaner } 1.44 \mathrm{mg} / \mathrm{kg} \text {, praziquantel } 10.0 \mathrm{mg} / \mathrm{kg} \text {, eprinomectin } 0.5 \mathrm{mg} / \mathrm{kg} \text {. } \\
2 \text { Esafoxolaner alone } 1.44 \mathrm{mg} / \mathrm{kg} \text {. } \\
{ }^{3} \text { Fipronil } 10.0 \mathrm{mg} / \mathrm{kg} \text {, (S)-methoprene } 12.0 \mathrm{mg} / \mathrm{kg} \text {, praziquantel } 10.0 \mathrm{mg} / \mathrm{kg} \text {, eprinomectin } 0.5 \mathrm{mg} / \mathrm{kg} \text {. } \\
{ }^{4} \text { Esafoxolaner } 0.72 \mathrm{mg} / \mathrm{kg} \text {, praziquantel } 5.0 \mathrm{mg} / \mathrm{kg} \text {, eprinomectin } 0.25 \mathrm{mg} / \mathrm{kg} \text {. } \\
{ }^{5} \text { Esafoxolaner } 2.88 \mathrm{mg} / \mathrm{kg} \text {, praziquantel } 20.0 \mathrm{mg} / \mathrm{kg} \text {, eprinomectin } 1.0 \mathrm{mg} / \mathrm{kg} \text {. } \\
{ }^{6} \mathrm{n}=\text { number of cats per group (equal number of males and females). }\end{array}$} \\
\hline
\end{tabular}

treatment administration. Equal numbers of males and females were randomly allocated to treatment groups based on pre-treatment body weight within sex. Cats were housed indoors, in an environmental-controlled facility, and were group housed by sex and treatment-group, except during the 4 days following treatment when they were individually housed to avoid treatment cross-contamination and to allow individual monitoring. All cats were observed 2 and $4 \mathrm{~h}$ after treatment application and once daily throughout the study, for the monitoring of health abnormalities and adverse reactions.

\section{Treatments and blood samplings}

All topical treatments were applied on one spot directly on the skin, after parting the hair, in the midline of the neck between the base of the skull and the shoulder blades. The intra-venous injection was given in the cephalic vein.

All blood samples were collected into lithium heparin tubes and processed into plasma by centrifugation. For animal welfare purposes, the individual blood sample volumes were limited to avoid exceeding cumulative blood withdrawal of approximately $15 \%$ of circulating volume in 4 weeks.

\section{Study designs}

Designs of Studies \#1 and \#2 are summarized in Table 1.

\section{Study \#1}

This study was designed to evaluate the main pharmacokinetic parameters $\left(C_{\max }, T_{\max }, C_{\text {last }}, T_{\text {last }}\right.$, half-life, AUC, bioavailability) of the three compounds administered topically once in the novel formulation at the recommended minimum dose, and the non-interference of the three compounds between each other. A comparison with Broadline ${ }^{\circledR}$, a topical endectoparasiticide product for cats in which eprinomectin and praziquantel are present in identical concentrations, was also used for analysis of non-interference in relation to the two endoparasiticide compounds.

Group 1 was treated topically with NexGard ${ }^{\circledR}$ Combo at the minimum recommended dose for analysis of the plasma profile of esafoxolaner, eprinomectin and praziquantel. Group 2 was treated topically with esafoxolaner alone, formulated with identical solvents/excipients as those of the novel formulation, for non-interference evaluation of esafoxolaner. Group 3 was treated with Broadline ${ }^{\circledR}$ for comparison of plasma levels of eprinomectin and praziquantel, and partial bridging of pharmacokinetic data from Broadline ${ }^{\circledR}$ for non-interference evaluation of eprinomectin and praziquantel in the novel formulation. Group 4 was treated intravenously with esafoxolaner for bioavailability calculation of topical esafoxolaner in the novel formulation.

Blood samples were collected prior to treatment and over a period of 70 days after treatment as detailed in Table 1. 


\section{Study \#2}

This study was designed to evaluate the dose proportionality/linearity of esafoxolaner, eprinomectin and praziquantel, after a single topical administration of the novel formulation at different doses, and to evaluate accumulation and time to steady state of the three active ingredients following repeated topical administrations.

Groups 1, 2 and 3, the dose proportionality/linearity groups, were treated once topically with $0.5,1$ or 2 times the minimum recommended dose volume of NexGard ${ }^{\circledR}$ Combo, respectively. Blood samples were collected prior to treatment and over a period of 91 days after the treatment, as detailed in Table 1.

Group 4, the accumulation and time to steady state group, was treated five times at 28-day intervals, topically with NexGard ${ }^{\circledR}$ Combo at the minimum recommended dose. In this group, blood samples were collected prior to each treatment, during the 4 weeks following each treatment, and for 8 weeks after the last treatment, as detailed in Table 1.

\section{Plasma analysis of esafoxolaner, eprinomectin and praziquantel}

Esafoxolaner, eprinomectin and praziquantel were quantitatively analyzed using liquid chromatography coupled to tandem mass spectrometry (LC-MS/MS). The cat plasma samples and internal standards were subjected to semi-automated solid phase extraction in a 96-well plate format followed by reversed-phase HPLC with gradient elution. The extracted analytes were quantified by an AB Sciex API 5000 mass spectrometer system using an electrospray interface. Drugs and internal standards were detected in positive ionization mode using multiple reaction monitoring of the precursor to product ions transition of $\mathrm{m} / \mathrm{z} 626.2 \rightarrow 470.3,313.3 \rightarrow 203.2$, and $914.5 \rightarrow 186.2$ for esafoxolaner, praziquantel, and eprinomectin B1a, respectively. Chromatograms were integrated by the Analyst ${ }^{\circledR}$ 1.6.2 software and peak areas were determined.

The validated lower and upper limits of quantitation were 0.2 and $100 \mathrm{ng} / \mathrm{mL}$, respectively. Sample concentrations were determined based on a fortified matrix calibration curve ranging from 0.2 to $100 \mathrm{ng} / \mathrm{mL}$ for each analyte with quality control samples, control plasma samples, and acceptance criteria according to EMA and FDA guidelines [4, 7]. Repeatability (precision), accuracy, assay specificity, dilution, recovery and matrix effect, carryover, stability in plasma and all solutions was established.

\section{Pharmacokinetics analysis}

Pharmacokinetic (PK) parameters were calculated from individual plasma concentrations using the non-compartmental analysis function of WinNonlin ${ }^{\circledR}$ software (Phoenix 64 Build, version 6.3). The non-compartmental approach was consistent with the extravascular (topical) or IV bolus route of administration.

The peak drug plasma concentration $C_{\max }$ and the time from dosing to the maximum concentration values $T_{\max }$ were obtained directly from plasma concentration versus time data for each cat and then averaged for each topical treatment group.
The terminal elimination phase was determined using at least the final three observed concentrations and not including $C_{\max }$. The first order rate constant, $\lambda_{\mathrm{z}}$, associated with the terminal log-linear portion of the curve was estimated via linear regression of the log plasma concentration versus time curve. The terminal plasma half-life using $\ln (2) / \lambda_{\mathrm{z}}$ was calculated for each animal, and then averaged for each treatment group. The area under the concentration versus time curve (AUC) from the time of dosing to the time to the last quantifiable concentration $\left(\mathrm{AUC}_{0-\text { Tlast }}\right)$ was calculated using the linear up, log down trapezoidal method, and extrapolated to infinity $\left(\mathrm{AUC}_{0-\mathrm{inf}}\right)$ using the formula $\mathrm{AUC}_{0-\text { Tlast }}+C_{\text {last }} / \lambda_{\mathrm{z}}$.

Bioavailability $(\% \mathrm{~F})$ of esafoxolaner in the NexGard Combo treated group was determined as the mean dose-normalized $\mathrm{AUC}_{0-\text { inf }}$ (topical)/AUC ${ }_{0-\text { inf }}$ (IV) for the active substance. Bioavailability of eprinomectin and praziquantel was determined in other studies. The systemic clearance of esafoxolaner in plasma (Cls) defined as dose/ $\mathrm{AUC}_{0-\text { inf }}$, and the volume of distribution at steady state $\left(V_{\mathrm{ss}}\right)$, which is $\mathrm{AUMC}_{0-\text { inf }} /$ $\mathrm{AUC}_{0-\text { inf }} \times \mathrm{Cls}$, was calculated from WinNonlin ${ }^{\circledR}$ for the IV esafoxolaner group only. Due to the long terminal plasma half-life of esafoxolaner, each cat was not used as its own control and bioavailability was calculated on the average. In order to determine and compare the PK parameters of eprinomectin and praziquantel when administered topically with esafoxolaner in NexGard ${ }^{\circledR}$ Combo or within Broadline ${ }^{\circledR}$, statistical evaluations of the PK parameters for different formulations were performed using a paired student's $t$-test and a $p$-value $<0.05$ was used as the criterion for difference.

Dose proportionality for the three active ingredients was assessed by calculating the linear relationship between AUC or $C_{\max }$ and the dose using the simple linear regression model.

Accumulation ratios were calculated using $C_{\max }$ and AUC for the three active ingredients after the 1st and 5th dose following five topical doses of the novel formulation. Furthermore, steady state was assessed using paired student's $t$-test on esafoxolaner, eprinomectin and praziquantel concentrations determined immediately prior to each dose of the novel formulation ( $p$-values result from a comparison of two consecutive doses).

\section{Results}

\section{Pharmacokinetics}

Pharmacokinetic parameters of esafoxolaner administered topically in the novel formulation, topically alone, intravenously alone, and of eprinomectin and praziquantel administered topically in the novel formulation and in Broadline ${ }^{\circledR}$ are presented in Table 2.

\section{Esafoxolaner}

The esafoxolaner average concentration-time curves are plotted in Figure 1.

After a single topical application of the combined formulation at the minimum recommended dose, esafoxolaner (applied at $1.44 \mathrm{mg} / \mathrm{kg}$ ) increased up to a mean $C_{\max }$ of $130 \pm 36 \mathrm{ng} / \mathrm{mL}$, reached at a mean $T_{\max }$ of $7.13 \pm 3.1$ days (the mean esafoxolaner concentration $24 \mathrm{~h}$ after treatment was 
Table 2. Summary of the pharamacokinetic parameters (mean $\pm S D$ ) of esafoxolaner, eprinomectin and praziquantel in NexGard ${ }^{\circledR}$ Combo following a single topical application at the minimum recommended dose, of eprinomectin and praziquantel in Broadline ${ }^{\circledR}$, and of esafoxolaner alone administered topically or intravenously.

\begin{tabular}{|c|c|c|c|c|c|c|c|c|}
\hline Analyte & Group $^{1}$ & $\begin{array}{r}T_{1 / 2} \\
\text { (day) }\end{array}$ & $\begin{array}{l}T_{\max } \\
\text { (day) }\end{array}$ & $\begin{array}{c}C_{\max } \\
(\mathrm{ng} / \mathrm{mL})\end{array}$ & $\begin{array}{c}\mathrm{AUC}_{0-\text { Tlast }} \\
(\text { day } \times \mathrm{ng} / \mathrm{mL})\end{array}$ & $\begin{array}{c}\mathrm{AUC}_{0-\mathrm{inf}} \\
(\text { day } \times \mathrm{ng} / \mathrm{mL})\end{array}$ & $\begin{array}{c}\mathrm{Cl} \\
(\mathrm{mL} / \mathrm{day} / \mathrm{kg})\end{array}$ & $\begin{array}{c}V_{\mathrm{ss}} \\
(\mathrm{mL} / \mathrm{kg})\end{array}$ \\
\hline \multirow[t]{3}{*}{ Esafoxolaner -Topical } & 1 & $21.7 \pm 2.8$ & $7.13 \pm 3.1$ & $130 \pm 36$ & $4411 \pm 1525$ & $4972 \pm 1711$ & NA & NA \\
\hline & 2 & $20.8 \pm 6.4$ & $3.75 \pm 3.1$ & $218 \pm 68$ & $6368 \pm 1665$ & $7181 \pm 2250$ & NA & NA \\
\hline & $p$-value ${ }^{2}$ & 0.713 & 0.019 & 0.0305 & 0.0792 & 0.0923 & NA & NA \\
\hline \multirow[t]{3}{*}{ Eprinomectin -Topical } & 1 & $5.42 \pm 2.7$ & $1.46 \pm 0.47$ & $23.6 \pm 11$ & $156 \pm 94$ & $159 \pm 94$ & NA & NA \\
\hline & 3 & $5.63 \pm 1.6$ & $1.71 \pm 0.97$ & $27.1 \pm 16$ & $175 \pm 67$ & $179 \pm 69$ & NA & NA \\
\hline & $p$-value ${ }^{2}$ & 0.806 & 0.476 & 0.6 & 0.656 & 0.642 & NA & NA \\
\hline \multirow[t]{3}{*}{ Praziquantel - Topical } & 1 & $4.30 \pm 1.9$ & $0.292 \pm 0.08$ & $107 \pm 59$ & $123 \pm 25$ & $132 \pm 23$ & NA & NA \\
\hline & 3 & $3.50 \pm 2.2$ & $0.313 \pm 0.06$ & $118 \pm 88$ & $185 \pm 93$ & $173 \pm 89$ & $\mathrm{~N}$ & NA \\
\hline & $p$-values ${ }^{2}$ & 0.462 & 0.351 & 0.797 & 0.0897 & 0.187 & NA & NA \\
\hline \multirow[t]{3}{*}{ Esafoxolaner -Intravenous } & 4 & $21.0 \pm 4.2$ & NA & NA & $9586 \pm 1985$ & $10,542 \pm 2029$ & $141 \pm 29.5$ & $4016 \pm 1190$ \\
\hline & $p$-value ${ }^{2}$ vs. 1 & 0.657 & NA & NA & NA & NA & NA & NA \\
\hline & $p$-value ${ }^{2}$ vs. 2 & 0.484 & NA & NA & NA & NA & NA & NA \\
\hline
\end{tabular}

$T_{1 / 2}=$ plasma half-life; $T_{\max }=$ time from dosing to the maximum concentration; $C_{\max }=$ peak drug plasma concentration; AUC $=$ area under the concentration versus time curve: $0-$ Tlast $=$ from the time of dosing to the time to the last quantifiable concentration, $0-$ inf $=$ from the time of dosing to infinity (by extrapolation); $\mathrm{Cl}=$ systemic clearance; $V_{\mathrm{ss}}=$ volume of distribution at steady-state.

${ }^{1}$ Group 1: NexGard Combo - Topical; esafoxolaner $1.44 \mathrm{mg} / \mathrm{kg}$, eprinomectin $0.5 \mathrm{mg} / \mathrm{kg}$, praziquantel $10.0 \mathrm{mg} / \mathrm{kg}$.

Group 2: Esafoxolaner alone - Topical; esafoxolaner $1.44 \mathrm{mg} / \mathrm{kg}$.

Group 3: Broadline ${ }^{\circledR}$ - Topical; fipronil $10.0 \mathrm{mg} / \mathrm{kg}$, (S)-methoprene $12.0 \mathrm{mg} / \mathrm{kg}$, eprinomectin $0.5 \mathrm{mg} / \mathrm{kg}$, praziquantel $10.0 \mathrm{mg} / \mathrm{kg}$.

Group 4: Esafoxolaner - IV; esafoxolaner $1.44 \mathrm{mg} / \mathrm{kg}$.

${ }^{2}$ Paired student's $t$-test.

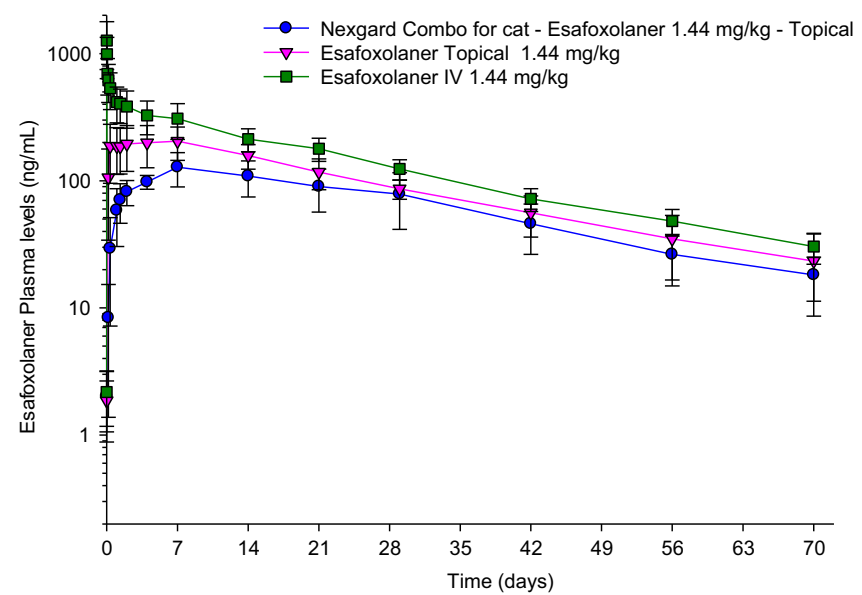

Figure 1. Esafoxolaner average concentration-time curves, when administered in NexGard ${ }^{\circledR}$ Combo, or alone in a topical or in an intravenous formulation.

$58.6 \pm 28.2 \mathrm{ng} / \mathrm{mL})$. Concentrations then declined steadily and were quantifiable for at least 10 weeks with mean concentration of $18.2 \pm 6.9 \mathrm{ng} / \mathrm{mL} 70$ days after dosing. Mean $\mathrm{AUC}_{0-\text { Tlast }}$ was $4411 \pm 1525$ day $\times \mathrm{ng} / \mathrm{mL}$, and the individual plasma half-lives ranged from 19.4 to 27.8 days (mean = $21.7 \pm 2.8$ days). The full pharmacokinetic curve was captured and less than $20 \%$ of the AUC was extrapolated for all animals.

The comparison of esafoxolaner administered topically in the novel formulation (combined with eprinomectin and praziquantel), and administered topically alone, showed no significant difference for $T_{1 / 2}, C_{\text {last }}$ and AUCs $\left(\mathrm{AUC}_{0-\text { Tlast }}\right.$ and $\left.\mathrm{AUC}_{0-\text { inf }}\right)(p \leq 0.08)$, and a significant difference for $C_{\max }$ and $T_{\max }(p=0.031$ and $p=0.019$, respectively).
Intravenous administration of esafoxolaner at $1.44 \mathrm{mg} / \mathrm{kg}$ revealed a mean half-life of $21.0 \pm 4.2$ days (ranging from 14.8 to 26.3 days), similar to values obtained after topical application in the combined formulation or alone $(p=0.657$ and 0.484 , respectively), indicating that the topical terminal plasma half-life was not absorption-limited. The clearance and volume of distribution were $141 \pm 29.5 \mathrm{~mL} / \mathrm{day} / \mathrm{kg}$ and $4016 \pm 1190 \mathrm{~mL} / \mathrm{kg}$, respectively. Topical esafoxolaner in the novel formulation was absorbed with an absolute bioavailability of $47.2 \%$.

\section{Praziquantel}

The praziquantel average concentration-time curves are plotted in Figure 2.

After a single topical administration of NexGard ${ }^{\circledR}$ Combo, praziquantel concentrations peaked quickly, indicating rapid absorption. The maximum concentrations were reached in 4 to $8 \mathrm{~h}$; the mean $C_{\max }$ was $107 \pm 59 \mathrm{ng} / \mathrm{mL}$. Concentrations then declined steadily with the last quantifiable plasma concentrations reached between 7 and 42 days following treatment. The mean half-life was $4.3 \pm 1.9$ days and the mean $\mathrm{AUC}_{0-\text { Tlast }}$ was $123 \pm 25$ day $\times \mathrm{ng} / \mathrm{mL}$. The full pharmacokinetic curve was captured and less than $20 \%$ of the AUC was extrapolated for all animals. When administered in Broadline ${ }^{\circledR}$, all PK parameters of praziquantel were not significantly different to those of the novel formulation. The bioavailability of praziquantel had been determined in the Broadline formulation to be $45 \%$ [16].

\section{Eprinomectin}

The eprinomectin average concentration-time curves are plotted in Figure 3. 


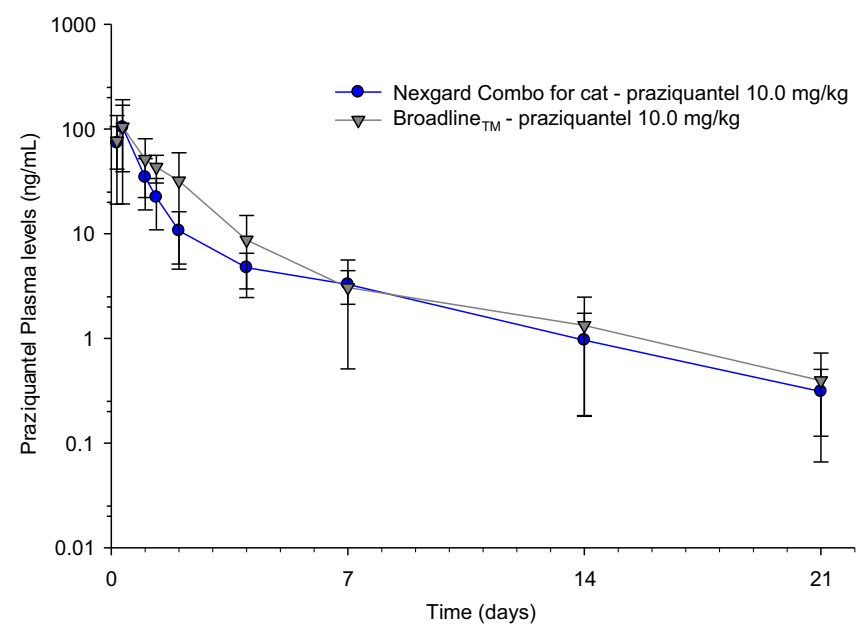

Figure 2. Praziquantel average concentration-time curves, when administered in NexGard ${ }^{\circledR}$ Combo, or in Broadline ${ }^{\circledR}$.

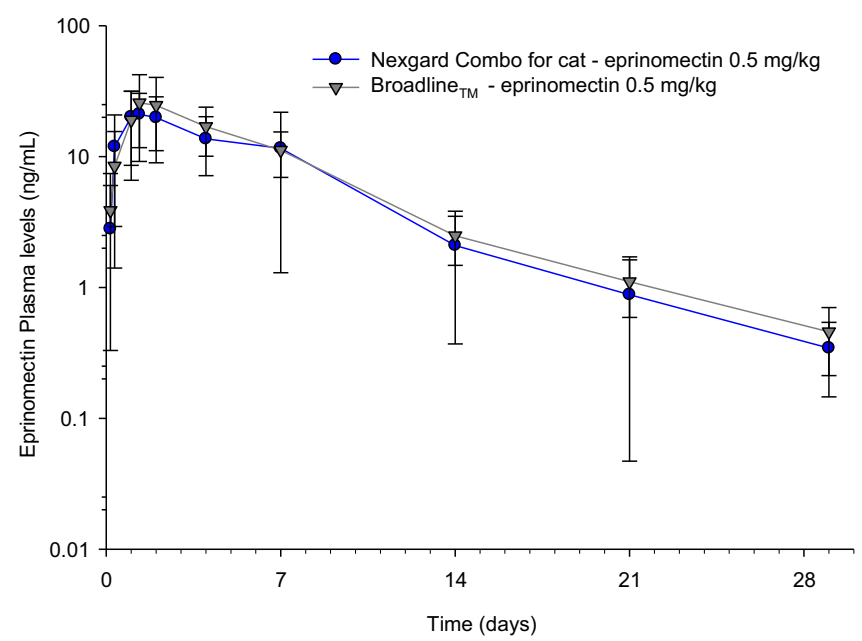

Figure 3. Eprinomectin average concentration-time curves, when administered in NexGard ${ }^{\circledR}$ Combo, or in Broadline ${ }^{\circledR}$.

After a single topical administration of NexGard ${ }^{\circledR}$ Combo, for eprinomectin, a mean $C_{\max }$ of $23.6 \pm 11.0 \mathrm{ng} / \mathrm{mL}$ was achieved at a mean $T_{\max }$ of $35 \mathrm{~h}$, and was followed by a gradual decrease in concentrations below the limit of quantitation after, on average, 28 days. The mean half-life was $5.4 \pm 2.7$ days and the mean $\mathrm{AUC}_{0-\text { Tlast }}$ was $156 \pm 94$ day $\times \mathrm{ng} / \mathrm{mL}$. The full pharmacokinetic curve was captured and less than $20 \%$ of the AUC was extrapolated for all animals. When administered in Broadline $^{\circledR}$, all PK parameters of eprinomectin were not significantly different to those of the novel formulation. The bioavailability of eprinomectin had been determined in the Broadline formulation to be $31 \%$ [16].

\section{Dose proportionality/linearity of the novel formulation}

Plots of mean $C_{\max }$ and $\mathrm{AUC}_{\text {last }}$ values as a function of increasing dose are shown in Figure 4.

After a single topical administration of three increasing doses of the combined formulation $(0.5 \times, 1 \times$ and $2 \times$ the intended minimum label dose), $\mathrm{C}_{\max }$ and $\mathrm{AUC}_{0 \text {-Tlast }}$ of the three active substances increased approximately proportionally with the dose, as illustrated in Table 3.

For esafoxolaner, praziquantel and eprinomectin, the $0.5 \times$ and the $2 \times$ groups, compared to the $1 \times$ group, had average $C_{\text {max }}$ and AUC ratios ranging from 0.4 to 0.6 and 1.9 to 2.9, respectively. These results indicate that the PK parameters of esafoxolaner, praziquantel and eprinomectin are dose-proportional over the dosing ranges of $0.72-2.88 \mathrm{mg} / \mathrm{kg}, 5-20 \mathrm{mg} /$ $\mathrm{kg}$, and $0.25-1.0 \mathrm{mg} / \mathrm{kg}$, i.e. $0.5 \times$ to $2 \times$ the minimum dose, respectively, following a single topical dose of $\mathrm{NexGard}^{\circledR}$ Combo.

\section{Multiple dose kinetics of the novel formulation}

Mean plasma concentrations of esafoxolaner, praziquantel and eprinomectin after five topical administrations of NexGard $^{\circledR}$ Combo at 4-week intervals are presented in Figure 5.

The AUC accumulation factor for esafoxolaner was 3.2 and the steady state was reached by the fifth 4 -weekly dose. The AUC accumulation factor for eprinomectin was 2.1 and the steady state was reached by the fifth 4 -weekly dose. No apparent accumulation (accumulation factor of $\sim 1$ ) was observed for praziquantel after five doses at 4-week intervals and the steady state was reached by the second dose.

\section{Tolerance}

None of the 62 cats included in both studies experienced any adverse reactions related to any of the treatments.

\section{Discussion}

The PK profile of esafoxolaner in NexGard ${ }^{\circledR}$ Combo was characterized by a long persistence, i.e. a quantifiable concentration lasting more than 13 weeks after a single administration at minimum dose due to high plasma protein binding, a high volume of distribution $\left(V_{\mathrm{ss}}=4.0 \mathrm{~L} / \mathrm{kg}\right)$, and a very low intrinsic clearance resulting in a long terminal half-life of 21 days. The $\mathrm{PK}$ profile of esafoxolaner in the combined formulation was in accordance with ectoparasiticide objectives of rapid onset and sustained efficacy of at least one month. The $\mathrm{EC}_{90}$ of esafoxolaner is $19.1 \pm 2.1 \mathrm{ng} / \mathrm{mL}$ for $C$. felis and $43.1 \mathrm{ng} / \mathrm{mL}$ for ticks (Ixodes scapularis) [internal Sponsor's data], and these efficacious plasma levels were maintained from $24 \mathrm{~h}$ until 6-8 weeks after a single application. This was confirmed by efficacy data obtained with NexGard ${ }^{\circledR}$ Combo against fleas $[34,37]$ and ticks $[28,36]$ in cats.

Eprinomectin and praziquantel plasma profiles following a topical application of the combined formulation have systemic endoparasiticide concentrations needed to kill nematodes and cestodes. Both compounds also have appropriate PK parameters (AUC, $C_{\max }$ and $T_{\max }$ ), that are identical to those of Broadline ${ }^{\circledR}$, a product with proven efficacy against nematodes and cestodes [12, 29, 35]. This was confirmed by efficacy data obtained with NexGard ${ }^{\circledR}$ Combo against both nematodes and cestodes [13] in cats.

Esafoxolaner did not interfere with the ADME properties of eprinomectin and praziquantel. The AUC, half-life and $C_{\text {last }}$, the most important parameters for sustained preventive flea and tick 

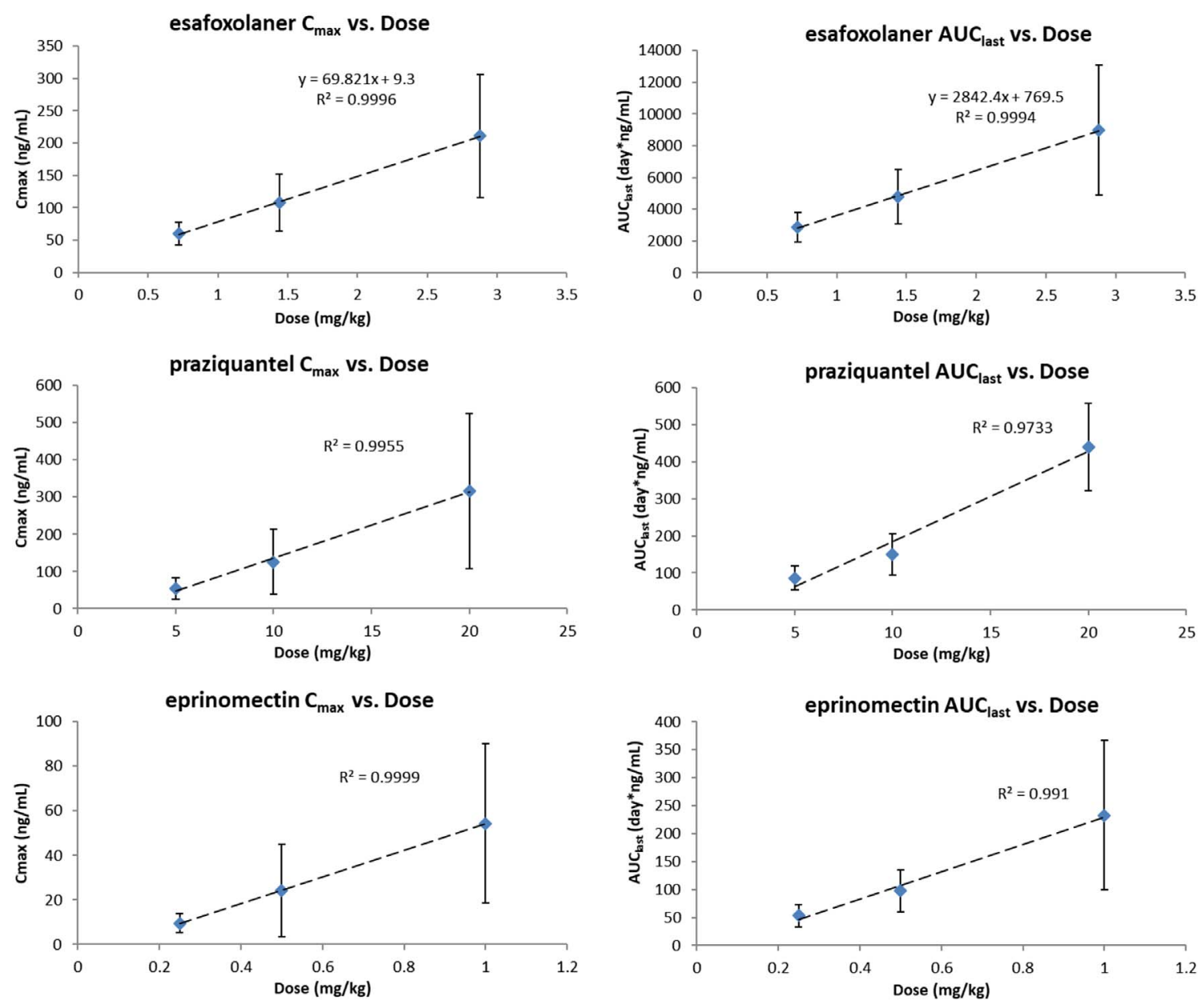

Figure 4. Plots of mean $\mathrm{C}_{\max }$ values and $\mathrm{AUC}_{\text {last }}$ values as a function of increasing dose for esafoxolaner, praziquantel and eprinomectin.

Table 3. Mean $C_{\max }$ and AUC for esafoxolaner, eprinomectin and praziquantel following a topical single administration 0.5 , 1 or 2 times the intended minimum dose of the novel formulation.

\begin{tabular}{|c|c|c|c|c|c|}
\hline Dose Level & Dose $(\mathrm{mg} / \mathrm{kg})$ & $C_{\max }(\mathrm{ng} / \mathrm{mL})$ & $\mathrm{AUC}_{0-\text { Tlast }}($ day $\times \mathrm{ng} / \mathrm{mL})$ & $C_{\max }$ Ratio & $\mathrm{AUC}_{0-\text { Tlast }}$ Ratio \\
\hline \multicolumn{6}{|l|}{ Esafoxolaner } \\
\hline $0.5 \times$ & 0.72 & $60.8 \pm 17.7$ & $2873 \pm 931$ & 0.6 & 0.6 \\
\hline $1 \times$ & 1.44 & $108 \pm 43.7$ & $4777 \pm 1714$ & - & - \\
\hline $2 \times$ & 2.88 & $211 \pm 94.8$ & $8984 \pm 4071$ & 2.0 & 1.9 \\
\hline \multicolumn{6}{|l|}{ Praziquantel } \\
\hline $0.5 \times$ & 5 & $54.7 \pm 28.8$ & $86.5 \pm 33.0$ & 0.5 & 0.6 \\
\hline $1 \times$ & 10 & $126 \pm 87.2$ & $150 \pm 56.1$ & - & - \\
\hline $2 \times$ & 20 & $317 \pm 209$ & $440 \pm 118$ & 2.5 & 2.9 \\
\hline \multicolumn{6}{|l|}{ Eprinomectin } \\
\hline $0.5 \times$ & 0.25 & $9.44 \pm 4.26$ & $53.6 \pm 19.9$ & 0.4 & 0.5 \\
\hline $1 \times$ & 0.5 & $24.1 \pm 20.8$ & $97.8 \pm 37.4$ & - & - \\
\hline $2 \times$ & 1 & $54.3 \pm 35.8$ & $233 \pm 133$ & 2.3 & 2.4 \\
\hline
\end{tabular}

$C_{\max }=$ peak drug plasma concentration; $\mathrm{AUC}_{0-\text { Tlast }}=$ area under the concentration versus time curve, from the time of dosing to the time to the last quantifiable concentration.

efficacy were unchanged when the active ingredient was given as a fixed combination or alone. However, the higher $C_{\max }$ achieved in less time (shorter $T_{\max }$ ) for esafoxolaner administered alone, indicated possible slower absorption of esafoxolaner when administered in the novel formulation (in combination with eprinomectin and praziquantel). Nevertheless, this has no impact on the onset of curative efficacy of NexGard ${ }^{\circledR}$ Combo, as consistently confirmed by 24 -hour and 48-hour high levels of efficacy after treatment against fleas and ticks, respectively [28, 34, 36]. Eprinomectin and praziquantel had similar plasma profiles after administration in Broadline $^{\circledR}$ or in NexGard ${ }^{\circledR}$ Combo, which allows the bridging 

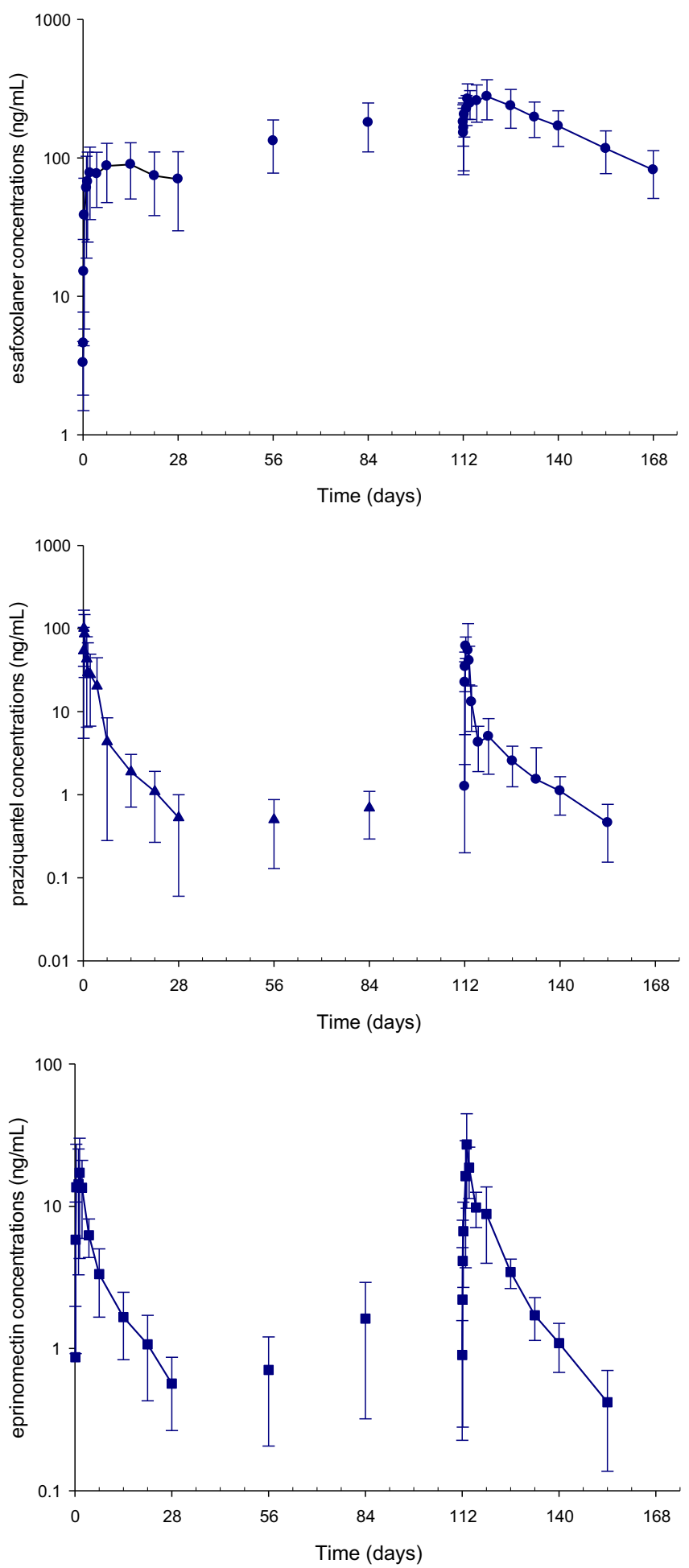

Figure 5. Mean plasma concentrations of esafoxolaner, praziquantel and eprinomectin after five topical administrations of NexGard ${ }^{\circledR}$ Combo at 4-week intervals.

of non-interference conclusions of these two compounds from the Broadline ${ }^{\circledR}$ studies [16].

Evaluations of plasma concentrations of the three compounds following administration of the novel formulation at half, equal, and double the minimum recommended dose demonstrated dose proportionality and thus linearity of ADME properties.

Evaluations of plasma concentrations of the three compounds following repeated topical treatments at 4-week intervals with the novel formulation demonstrated that a steady state was reached after five treatments for esafoxolaner and eprinomectin, and after two treatments for praziquantel. At steady state, the accumulation factor was 3.2 for esafoxolaner, 2.1 for eprinomectin and 1.0 (i.e. no accumulation) for praziquantel. Feline tolerance to the novel formulation was investigated in several target animal safety studies, namely two margin of safety studies [8]. In the first (pilot) study, two groups of cats were treated with the novel formulation at $3 \times$ and $5 \times$ multiples of the maximum exposure dose, four times at 2-week intervals, and one group was treated with a topical formulation of only esafoxolaner, twice at a 28 -day interval, providing $23 \times$ the maximum exposure dose of esafoxolaner in NexGard ${ }^{\circledR}$ Combo. In the second (regulatory) study, cats were treated with six repeated 4-week interval topical dosages of the novel formulation at $1 \times, 3 \times$, or $5 \times$ multiples of the maximum recommended dose. In the first study, no significant adverse reactions were seen in the novel formulation and in the esafoxolaner groups. In the regulatory study, no significant adverse reactions were seen at $1 \times$ and $3 \times$ overdoses, and one reversible neurological adverse reaction was seen at $5 \times$ overdose. This adverse reaction was attributed to eprinomectin, because of the nature of the signs typical of avermectin toxicity [8,23], and because of the good tolerance to a much higher dose of esafoxolaner in the first study. The results of these margin of safety studies support safe use of repeated treatments with the novel formulation and good tolerance of esafoxolaner and eprinomectin at accumulation factors of 3.2 and 2.1, respectively, which leaves long-term exposure well below the $5 \times$ maximum exposure dose.

NexGard ${ }^{\circledR}$ Combo offers a convenient and safe solution to veterinarians and pet owners for the treatment of multiparasitism in cats. Unlike the simultaneous use of narrow-spectrum parasiticide products, the use of a broad-spectrum endectoparasiticide product such as this novel formulation offers precise characterization and confirmations of safety and efficacy.

Acknowledgements. The authors gratefully acknowledge the staff at "Centre de Recherche de Saint-Vulbas, France" and "North Brunswick Research Center, USA" for conducting their respective study phases to high professional standards.

\section{Conflict of interest}

The work reported herein was funded by BoehringerIngelheim. The authors are current employees of BoehringerIngelheim Animal Heath. Other than that, the authors declare no conflict of interest. This document is provided for scientific purposes only. Any reference to a brand or trademark herein is for information purposes only and is not intended for any commercial purposes or to dilute the rights of the respective owners of the brand(s) or trademark(s).

$\operatorname{NexGard}^{\circledR}$ is a registered trademark of the BoehringerIngelheim Group. 


\section{References}

1. Beugnet F, Bourdeau P, Chalvet-Monfray K, Cozma V, Farkas R, Guillot J, Halos L, Joachim A, Losson B, Miró G, Otranto D, Renaud M, Rinaldi L. 2014. Parasites of domestic owned cats in Europe: Co-infestations and risk factors. Parasites \& Vectors, 7, 291.

2. Chai JY. 2013. Praziquantel treatment in trematode and cestode infections: An update. Infection and Chemotherapy, 45, 32-43.

3. Charles SD, Altreuther G, Reinemeyer CR, Buch J, Settje T, Cruthers L, Kok DJ, Bowman DD, Kazacos KR, Jenkins DJ, Schein E. 2005. Evaluation of the efficacy of emodepside + praziquantel topical solution against cestode (Dipylidium caninum, Taenia taeniaeformis, and Echinococcus multilocularis) infections in cats. Parasitology Research, 97, 33-40.

4. EMA (European Medicines Agency). 2012. Guideline on bioanalytical method validation. https://www.ema.europa.eu/ en/documents/scientific-guideline/guideline-bioanalytical-methodvalidation_en.pdf.

5. EMA (European Medicines Agency). 2019. Nexgard ${ }^{\circledR}$ summary of product characteristic (last update). https://www.ema.europa. eu/en/documents/product-information/nexgard-epar-productinformation_en.pdf.

6. EMA (European Medicines Agency). 2019. Nexgard Spectra ${ }^{\circledR}$ summary of product characteristics (last update). https://www. ema.europa.eu/en/documents/product-information/nexgard-spectra-epar-product-information_en.pdf.

7. FDA. 2018. Bioanalytical method validation guidance for industry. https://www.fda.gov/files/drugs/published/Bioanalytical-Method-Validation-Guidance-for-Industry.pdf.

8. Gupta A, Baker C, Wang H, Targa N, Pfefferkorn A, Tielemans E. 2021. Target animal safety evaluation of a novel topical combination of esafoxolaner, eprinomectin and praziquantel for cats. Parasite, 28, 18.

9. Halos L, Lebon W, Chalvet-Monfray K, Larsen D, Beugnet F. 2014. Immediate efficacy and persistent speed of kill of a novel oral formulation of afoxolaner $\left(\mathrm{NexGard}^{\circledR}\right)$ against induced infestations with Ixodes ricinus ticks. Parasites \& Vectors, 5, 452.

10. Hampel V, Knaus M, Schäfer J, Beugnet F, Rehbein S. 2018. Treatment of canine sarcoptic mange with afoxolaner $\left(\right.$ NexGard $\left.^{\circledR}\right)$ and afoxolaner plus milbemycin oxime (NexGard Spectra ${ }^{\circledR}$ ) chewable tablets: efficacy under field conditions in Portugal and Germany. Parasite, 25, 63.

11. Hunter JS, Dumont P, Chester TS, Young DR, Fourie JJ, Larsen DL. 2014. Evaluation of the curative and preventive efficacy of a single oral administration of afoxolaner against cat flea Ctenocephalides felis infestations on dogs. Veterinary Parasitology, 201, 2017-2211.

12. Knaus M, Abu-Madi MA, Ibarra-Velarde F, Kok DJ, Kusi I, Postoli R, Chester ST, Rosentel J, Alva R, Irwin J, Visser M, Winter R, Rehbein S. 2014. Efficacy of a novel topical fipronil, (S)-methoprene, eprinomectin and praziquantel combination against naturally acquired intestinal nematode and cestode infections in cats. Veterinary Parasitology, 202, 18-25.

13. Knaus M, Baker C, Alva R, Mitchell E, Irwin J, Shukullari E, Veliu A, Ibarra-Velarde F, Liebenberg J, Reinemeyer C, Tielemans E, Wakeland K, Johnson C. 2021. Efficacy of a novel topical combination of esafoxolaner, eprinomectin and praziquantel in cats against Toxocara cati and Dipylidium caninum. Parasite, 28, 28

14. Kondo Y, Kinoshita G, Drag M, Chester T, Larsen D. 2014. Evaluation of the efficacy of afoxolaner against Haemaphysalis longicornis on dogs. Veterinary Parasitology, 201, 229-231.

15. Kunkle B, Daly S, Dumont P, Drag M, Larsen D. 2014. Assessment of the efficacy of orally administered afoxolaner against Rhipicephalus sanguineus sensu lato. Veterinary Parasitology, 201, 226-228.

16. Kvaternick V, Kellermann M, Knaus M, Rehbein S, Rosentel J. 2014. Pharmacokinetics and metabolism of eprinomectin in cats when administered in a novel topical combination of fipronil, (S)-methoprene, eprinomectin and praziquantel. Veterinary Parasitology, 202, 2-9.

17. Lappin MR. 2018. Update on flea and tick associated diseases of cats. Veterinary Parasitology, 254, 26-29.

18. Lebon W, Beccati M, Bourdeau P, Brement T, Bruet V, Cekiera A, Crosaz O, Darmon C, Guillot J, Mosca M, Pin D, Popiel J, Pomorska Handwerker D, Larsen D, Tielemans E, Beugnet F, Halos L. 2018. Efficacy of two formulations of afoxolaner (NexGard and NexGard Spectra) for the treatment of generalized demodicosis in dogs, in veterinary dermatology referral centers in Europe. Parasites \& Vectors, 11, 506.

19. Letendre L, Harriman J, Drag M, Mullins A, Malinski T, Rehbein S. 2017. The intravenous and oral pharmacokinetics of afoxolaner and milbemycin oxime when used as a combination chewable parasiticide for dogs. Journal of Veterinary Pharmacology and Therapeutics, 40(1), 35-43.

20. Letendre L, Huang R, Kvaternick V, Harriman J, Soll M. 2014. The intravenous and oral pharmacokinetics of afoxolaner used as a monthly chewable antiparasitic for dogs. Veterinary Parasitology, 201, 190-197.

21. Lucio-Forster A, Bowman D. 2011. Prevalence of fecal-borne parasites detected by centrifugal flotation in feline samples from two shelters in upstate New York. Journal of Feline Medicine and Surgery, $13,300-303$.

22. Machado MA, Campos DR, Lopes NL, Barbieri Bastos IP, Botelho CB, Correia TR, Scott FB, Fernades JI. 2018. Efficacy of afoxolaner in the treatment of otodectic mange in naturally infested cats. Veterinary Parasitology, 256, 29-31.

23. Merola VM, Eubig PA. 2012. Toxicology of avermectins and milbemycins (macrocylic lactones) and the role of P-glycoprotein in dogs and cats. Veterinary Clinics of North America Small Animal Practice, 42(2), 313-333.

24. Mitchell E, McCall JS, Chester T, Larsen D. 2014. Efficacy of afoxolaner against Ixodes scapularis ticks in dogs. Veterinary Parasitology, 201, 223-225.

25. Mitchell E, Dorr P, Everett WR, Chester T, Larsen D. 2014. Efficacy of afoxolaner against Dermacentor variabilis ticks in dogs. Veterinary Parasitology, 201, 220-222.

26. Ozoe Y, Asahi M, Ozoe F, Nakahira K, Mita T. 2010. The antiparasitic isoxazoline A1443 is a potent blocker of insect ligand-gated chloride channels. Biochemical and Biophysical Research Communications, 391, 744-749.

27. Pitt SR, Langholff W, Eagleson JS, Rehbein S. 1997. The efficacy of eprinomectin against induced infections of immature (fourth larval stage) and adult parasites in cattle. Veterinary Parasitology, 73, 119-128.

28. Prullage J, Pfefferkorn A, Knaus M, Frost J, Mitchell E, Tielemans E. 2021. Efficacy of a novel topical combination of esafoxolaner, eprinomectin and praziquantel against Ixodes ricinus and Ixodes scapularis in cats. Parasite, 28, 23.

29. Rehbein S, Capári B, Duscher G, Keidane D, Kirkova Z, Petkevicius S, Rapti D, Wagner A, Wagner T, Chester ST, Rosentel J, Tielemans E, Visser M, Winter R, Kley K, Knaus M. 2014. Efficacy against nematode and cestode infections and safety of a novel topical fipronil, (S)-methoprene, eprinomectin and praziquantel combination product in domestic cats under field conditions in Europe. Veterinary Parasitology, 202, 10-17.

30. Rehbein S, de Vos C, Beugnet F, Carithers D, Fourie J. 2018. Efficacy of once-monthly doses of oral afoxolaner and afoxolaner/milbemycin oxime in a well-controlled study for 
the treatment of canine generalized demodicosis. Open Journal of Veterinary Medicine, 8(12), 250-265.

31. Shoop WL, Hartline EJ, Gould BR, Waddel ME, McDowell RG, Kinney JB, Lahm GP, Long JK, Xu M, Wagerle T, Jones GS, Dietrich RF, Cordova D, Schroeder ME, Rhoades DF, Benner EA, Confalone PN. 2014. Discovery and mode of action of afoxolaner, a new isoxazoline parasiticide for dogs. Veterinary Parasitology, 201, 79-189.

32. Schroeder I, Altreuther G, Schimmel A, Deplazes P, Kok DJ, Schnyder M, Krieger KJ. 2009. Efficacy of emodepside plus praziquantel tablets (Profender tablets for dogs) against mature and immature cestode infections in dogs. Parasitology Research, 105, 31-38.

33. Soll MD, Kunkle BN, Royer GC, Yazwinski TA, Baggott DG, Wehner TA, Yoon S, Cramer LG, Rehbein S. 2013. An eprinomectin extended-release injection formulation providing nematode control in cattle for up to 150 days. Veterinary Parasitology, 192, 313-320.

34. Tielemans E, Buellet P, Young D, Viljoen A, Liebenberg J, Prullage J. 2021. Efficacy of a novel topical combination of esafoxolaner, eprinomectin and praziquantel against adult cat flea Ctenocephalides felis and flea egg production in cats. Parasite, 28, 21.
35. Tielemans E, Manavella C, Visser M, Chester T, Rosentel J. 2014. Efficacy in cats of a novel topical combination of fipronil, (S)-methoprene, eprinomectin, praziquantel, against induced infestations of Echinococcus multilocularis. Veterinary Parasitology, 202, 26-29.

36. Tielemans E, Pfefferkorn A, Viljoen A. 2021. Efficacy of a novel topical combination of esafoxolaner, eprinomectin and praziquantel against Rhipicephalus sanguineus in cats. Parasite, $28,24$.

37. Tielemans E, Otsuki T, Cheesman T, Selmes F, Pfefferkorn A, Prullage J. 2021. Efficacy of a novel topical combination of esafoxolaner, eprinomectin and praziquantel against fleas in cats, under field conditions. Parasite, 28, 22.

38. Traversa D. 2012. Pet roundworms and hookworms: A continuing need for global worming. Parasites \& Vectors, 5, 91-110.

39. Vale N, Gouveia MJ, Rinaldi G, Brindley PJ, Gärtner F, Correia da Costa JM. 2017. Praziquantel for Schistosomiasis: Singledrug metabolism revisited, mode of action, and resistance. Antimicrobial Agents Chemotherapy, 61(5), e02582-16.

40. Woods DJ, Vaillancourt VA, Wendt JA, Meeus PF. 2011. Discovery and development of veterinary antiparasitic drugs: Past, present and future. Future Medicinal Chemistry, 3, 887-896.

Cite this article as: Jacquot V, Buellet $\mathrm{P}$, Letendre L, Tong W, Li H \& Tielemans E. 2021. Pharmacokinetics of a novel endectoparasiticide topical formulation for cats, combining esafoxolaner, eprinomectinand praziquantel. Parasite $28,19$.

\section{PARASTE}

An international open-access, peer-reviewed, online journal publishing high quality papers on all aspects of human and animal parasitology

Reviews, articles and short notes may be submitted. Fields include, but are not limited to: general, medical and veterinary parasitology; morphology, including ultrastructure; parasite systematics, including entomology, acarology, helminthology and protistology, and molecular analyses; molecular biology and biochemistry; immunology of parasitic diseases; host-parasite relationships; ecology and life history of parasites; epidemiology; therapeutics; new diagnostic tools.

All papers in Parasite are published in English. Manuscripts should have a broad interest and must not have been published or submitted elsewhere. No limit is imposed on the length of manuscripts.

Parasite (open-access) continues Parasite (print and online editions, 1994-2012) and Annales de Parasitologie Humaine et Comparée (1923-1993) and is the official journal of the Société Française de Parasitologie. 